\title{
GROTHENDIECK GROUPS OF ALGEBRAS WITH NILPOTENT ANNIHILATORS
}

\author{
MAURICE AUSLANDER AND IDUN REITEN
}

(Communicated by Donald S. Passman)

\begin{abstract}
Let $R$ be a commutative noetherian ring and $i: R \rightarrow \Lambda$ an $R$ algebra such that $\Lambda$ is a finitely generated $R$-module. Then the annihilator of $\Lambda$ in $R$ is nilpotent if and only if the cokernel of the induced map of Grothendieck groups $i^{*}: K_{0}(\bmod \Lambda) \rightarrow K_{0}(\bmod R)$ is a torsion group.
\end{abstract}

Let $k$ be an algebraically closed field, and let $S=k\left[\left[X_{1}, \ldots, X_{n}\right]\right]$ be the formal power series ring in $n$ variables over $k$. Let $G$ be a finite subgroup of $\operatorname{GL}(n, k)$. Hence $G$ acts as a group of $k$-automorphisms of $S$, and we denote by $R$ the fixed ring $R=S^{G}$. Denote by $K_{0}(\bmod R)$ the Grothendieck group of the category of finitely generated $R$-modules $\bmod R$ modulo exact sequences. In [1] we proved that $K_{0}(\bmod R)$ is finitely generated. Under the additional assumption that $G$ acts freely we proved that $K_{0}(\bmod R)$ is isomorphic to $Z[R] \amalg H$, where $H$ is a finite group and $[R]$ denotes the image of $R$ in $K_{0}(\bmod R)$. The motivation for this paper was to show that the assumption that $G$ acts freely is not necessary. The proof we give here is based on the proof given in the Bielefeld May 1985 conference on representation theory and singularity theory. A different proof in the case that $G$ is abelian has been given in [3]. Our desired result is an easy consequence of the following general result which is also of independent interest. For the rest of the paper $R$ denotes a commutative noetherian ring and $\Lambda$ an $R$-algebra via a fixed map $i: R \rightarrow \Lambda$ such that $\Lambda$ is a finitely generated $R$-module.

THEOREM. The annihilator of $\Lambda, \operatorname{ann}_{R} \Lambda$, is a nilpotent ideal in $R$ if and only if $\operatorname{Coker}\left(K_{0}(\bmod \Lambda) \stackrel{i^{*}}{\rightarrow} K_{0}(\bmod R)\right)$ is a torsion group.

This result is a direct consequence of the following two propositions.

Proposition 1. If $\operatorname{ann}_{R} \Lambda$ is nilpotent, then

$$
\operatorname{Coker}\left(K_{0}(\bmod \Lambda) \stackrel{i^{*}}{\rightarrow} K_{0}(\bmod R)\right)
$$

is torsion.

PROOF. Now, $a n_{R} \Lambda$ is nilpotent if and only if $\Lambda_{p} \neq 0$ for all prime ideals $p$ in $R$. From this characterization it follows easily that if $\mathfrak{A}$ is an ideal in $R$ and we consider the $R / \mathfrak{A}$-algebra $R / \mathfrak{A} \rightarrow \Lambda / \mathfrak{A} \Lambda$, then $\operatorname{ann}_{R / \mathfrak{A}} \Lambda / \mathfrak{A} \Lambda$ is nilpotent. Assume that the proposition is false. Then because $R$ is noetherian there is an ideal $\mathfrak{A}$ in $R$ such that $\operatorname{Coker}\left(K_{0}(\bmod \Lambda / \mathfrak{A} \Lambda) \rightarrow K_{0}(\bmod R / \mathfrak{A})\right)$ is not torsion, and

Received by the editors December 1, 1986 and, in revised form, March 30, 1987.

1980 Mathematics Subject Classification (1985 Revision). Primary 13D15; Secondary 19A49.

Written with partial support from NSF. 
$\operatorname{Coker}\left(K_{0}(\bmod \Lambda / b \Lambda) \rightarrow K_{0}(\bmod R / b)\right)$ is torsion for any ideal $\mathfrak{B}$ in $R$ properly containing $\mathfrak{A}$. We can clearly assume that $\mathfrak{A}$ is zero.

Consider for a minimal prime ideal $p$ in $R$ the commutative exact diagram [2, p. 642]

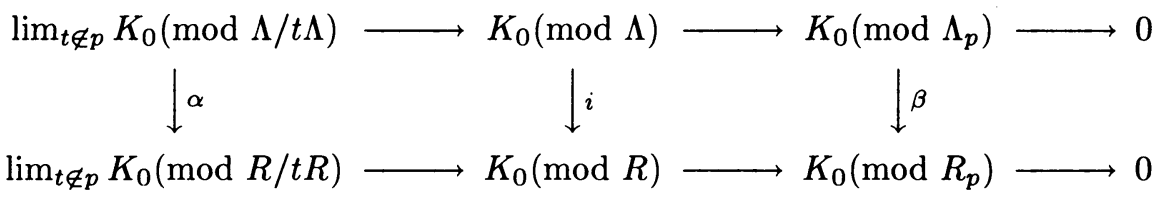

The snake lemma gives the exact sequence Coker $\alpha \rightarrow$ Coker $i^{*} \rightarrow$ Coker $\beta \rightarrow$ 0 . Since $R_{p}$ is a local artin $\operatorname{ring}, K_{0}\left(\bmod R_{p}\right) \simeq Z$, and since $\Lambda_{p} \neq 0, \beta$ is not zero, so that $\operatorname{Coker} \beta$ is torsion. By our assumption $\operatorname{Coker}\left(K_{0}(\bmod \Lambda / t \Lambda) \rightarrow\right.$ $\left.K_{0}(\bmod R / t R)\right)$ is torsion since $t \neq 0$, and hence Coker $\alpha$ is torsion. From this it follows that Coker $i^{*}$ is torsion, which contradicts our assumption, and the proof is done.

PROPOSITION 2. We have $\operatorname{rank}\left(\operatorname{Coker} K_{0}\left(\bmod \Lambda \stackrel{i^{*}}{\rightarrow} K_{0}(\bmod R)\right) \geq n\right.$, where $n$ denotes the number of minimal primes in $R$ not containing $\mathfrak{A}=\operatorname{ann}_{R} \Lambda$.

PROOF. Let $p_{1}, \ldots, p_{n}, \ldots, p_{r}$ be the minimal primes in $R$, where $\mathfrak{A} \subsetneq p_{i}$ for $1 \leq i \leq n$ and $\mathfrak{A} \subset p_{i}$ for $n<i \leq r$. Let $T=R \backslash \bigcup_{i=1}^{r} p_{i}$, and consider the diagram

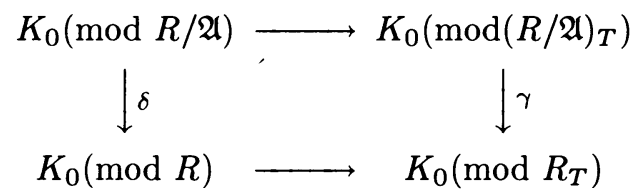

Since $R_{T}$ is artin, $K_{0}\left(\bmod R_{T}\right)$ is a free group with basis $\left\{\left[\left(R / p_{i}\right)_{T}\right] ; 1 \leq i \leq r\right\}$ and since $(R / \mathfrak{A})_{T}$ is artin $K_{0}\left(\bmod (R / \mathfrak{A})_{T}\right)$ is a free group with basis $\left\{\left[\left(R / p_{i}\right)_{T}\right] ; r \leq\right.$ $i \leq r\}$. Hence we have rank Coker $\delta \geq \operatorname{rank}$ Coker $\gamma \geq n$. Since $i: R \rightarrow \Lambda$ has the factorization $R \rightarrow R / \mathfrak{A} \rightarrow \Lambda$, we get rank Coker $i^{*} \geq \operatorname{rank}$ Coker $\delta \geq n$.

We end this note with the following consequences of Proposition 1 .

COROLlARY 3. If $\Lambda$ is a commutative semilocal regular domain and $R \subset \Lambda$, then $\operatorname{rank} K_{0}(\bmod R)=1$.

ProOF. Since $\Lambda$ is regular, $K_{0}(\bmod \Lambda) \simeq K_{0}(\mathscr{P}(\Lambda), 0)$, where $\mathscr{P}$ denotes the category of finite generated projective $\Lambda$-modules and $K_{0}(\mathscr{P}, 0)$ denotes the free group on the isomorphism classes of objects in $\mathscr{P}$ modulo split exact sequences. Then $K_{0}(\bmod \Lambda) \simeq Z$ since every finitely generated projective $\Lambda$-module is free. Since $R \subset \Lambda, R$ is also a domain. If $L$ denotes the quotient field of $R$, we have a surjection $K_{0}(\bmod R) \rightarrow K_{0}(\bmod L)$, so that $\operatorname{rank} K_{0}(\bmod R) \geq 1$. It then follows from Proposition 1 that $\operatorname{rank} K_{0}(\bmod R)=1$.

COROLlARY 4. Let $\Lambda$ be a commutative complete regular local domain and $G$ a finite group acting on $\Lambda$ as ring automorphisms, such that the order of $G$ is invertible in $\Lambda$. Then $K_{0}\left(\bmod \Lambda^{G}\right) \simeq Z \oplus H$, where $H$ is a finite group.

PROOF. $\Lambda$ is a finitely generated $\Lambda^{G}$-module $[\mathbf{4}$, Corollary 5.9$]$ and $R=\Lambda^{G}$ is noetherian $\left[4\right.$, Corollary 1.12]. Then rank $K_{0}\left(\bmod \Lambda^{G}\right)=1$ follows from Corollary 3 , and it only remains to show that $K_{0}\left(\bmod \Lambda^{G}\right)$ is finitely generated. This 
follows as in [1, Proposition 3.4]: Since $\operatorname{End}_{\Lambda G}(\Lambda) \simeq \Lambda^{G}$, there is a surjection $K_{0}(\bmod \Lambda G) \rightarrow K_{0}\left(\bmod \Lambda^{G}\right)$. Further $\Lambda G$ has finite global dimension since the order of $G$ is invertible in $\Lambda$, and hence $K_{0}(\bmod \Lambda G) \simeq K_{0}(\mathscr{P}(\Lambda G), 0)$. Since by our assumption on $\Lambda$ the Krull-Schmidt property holds for $\Lambda G, K_{0}(\mathscr{P}(\Lambda G), 0)$ is finitely generated.

\section{REFERENCES}

1. M. Auslander and I. Reiten, Grothendieck groups of algebras and orders, J. Pure Appl. Algebra 29 (1986), 1-51.

2. H. Bass, Algebraic K-theory, Benjamin, New York, 1968.

3. J. Herzog and H. Sanders, The Grothendieck group of invariant rings and of simple hypersurface singularities,

4. S. Montgomery, Fixed rings of finite automorphism groups of associative rings, Lecture Notes in Math., vol. 818, Springer-Verlag, Berlin and New York, 1980.

Department of Mathematics, Virginia Polytechnic Institute and State UNIVERSITY, BLACKSBURG, VIRGINIA 24061-4097

Current address (Maurice Auslander): Department of Mathematics, Brandeis University, Waltham, Massachusetts 02254

Current address (Idun Reiten): Department of Mathematics, University of Trondheim, 7055 Dragvoll, Norway 